\title{
Inappropriate Expression of an NLP Effector in Colletotrichum orbiculare Impairs Infection on Cucurbitaceae Cultivars via Plant Recognition of the C-Terminal Region
}

\author{
Nur Sabrina Ahmad Azmi, ${ }^{1}$ Suthitar Singkaravanit-Ogawa, ${ }^{1}$ Kyoko Ikeda, ${ }^{1}$ Saeko Kitakura, ${ }^{1}$ \\ Yoshihiro Inoue, ${ }^{1}$ Yoshihiro Narusaka, ${ }^{2}$ Ken Shirasu, ${ }^{3}$ Masanori Kaido, ${ }^{1}$ Kazuyuki Mise, ${ }^{1}$ and \\ Yoshitaka Takano ${ }^{1, \dagger}$ \\ ${ }^{1}$ Graduate School of Agriculture, Kyoto University, Kyoto, Japan; ${ }^{2}$ Research Institute for Biological Sciences Okayama, \\ Okayama, Japan; and ${ }^{3}$ RIKEN Center for Sustainable Resource Science, Yokohama, Japan
}

Accepted 9 September 2017.

\begin{abstract}
The hemibiotrophic pathogen Colletotrichum orbiculare preferentially expresses a necrosis and ethylene-inducing peptide 1 (Nep1)-like protein named NLP1 during the switch to necrotrophy. Here, we report that the constitutive expression of NLP1 in C. orbiculare blocks pathogen infection in multiple Cucurbitaceae cultivars via their enhanced defense responses. NLP1 has a cytotoxic activity that induces cell death in Nicotiana benthamiana. However, $C$. orbiculare transgenic lines constitutively expressing a mutant NLP1 lacking the cytotoxic activity still failed to infect cucumber, indicating no clear relationship between cytotoxic activity and the NLP1-dependent enhanced defense. NLP1 also possesses the microbe-associated molecular pattern (MAMP) sequence called nlp24, recognized by Arabidopsis thaliana at its central region, similar to NLPs of other pathogens. Surprisingly, inappropriate expression of a mutant NLP1 lacking the MAMP signature is also effective for blocking pathogen infection, uncoupling the infection block from the corresponding MAMP. Notably, the deletion analyses of NLP1 suggested that the C-terminal region of NLP1 is critical to enhance defense in cucumber. The expression of mCherry fused with the C-terminal 32 amino acids of NLP1 was enough to trigger the defense of cucurbits, revealing that the $\mathrm{C}$-terminal region of the NLP1 protein is recognized by cucurbits and, then, terminates $C$. orbiculare infection.
\end{abstract}

Necrosis and ethylene-inducing peptide 1 (Nep1) was originally discovered in the culture filtrate of Fusarium oxysporum as a secreted cytotoxic protein that causes cell death concomitant with ethylene production (Bailey 1995). Nep1-like proteins (NLPs) constitute a superfamily of proteins that are produced by various phytopathogenic prokaryotic and eukaryotic microorganisms (Gijzen and Nürnberger 2006; Oome and Van den Ackerveken 2014). Tertiary structural analyses on NLPs of Pythium aphanidermatum and Phytophthora parasitica revealed

${ }^{\dagger}$ Corresponding author: Yoshitaka Takano;

E-mail: ytakano@kais.kyoto-u.ac.jp

*The $\boldsymbol{e}$-Xtra logo stands for "electronic extra" and indicates that seven supplementary figures, one supplementary table, and supplementary materials and methods are published online.

(c) 2018 The American Phytopathological Society structural conservation of the NLPs with cytolytic and poreforming actinoporins of marine organisms (Ottmann et al. 2009). This data suggests that NLPs destabilize plasma membranes of plants, thereby resulting in plant cell death.

Interestingly, NLPs are also known to stimulate defense responses in dicotyledonous but not in monocotyledonous plants (Boller and Felix 2009; Gijzen and Nürnberger 2006; Qutob et al. 2006; Staats et al. 2007). Thus, NLPs are likely to have dual functions in plant-pathogenic microorganism interactions, i.e., triggering immune responses and acting as toxin-like virulence factors. Cytotoxic NLPs activate immunity-related gene expression in Arabidopsis thaliana, which clearly overlaps with that induced by flg22 (Bae et al. 2006; Qutob et al. 2006), suggesting the similarity of cytotoxic NLP-triggered defense to the microbeassociated molecular pattern (MAMP)-triggered immunity. Furthermore, whereas the oomycete pathogen Hyaloperonospora arabidopsidis produces multiple NLPs, all $H$. arabidopsidis NLPs lack the ability to cause necrosis in dicot plants, including the host Arabidopsis thaliana (Cabral et al. 2012), but can induce defense responses such as $P R I$ expression in A. thaliana (Oome et al. 2014). These suggest at least partial uncoupling of NLP-triggered plant defense activation from their cytotoxicity in A. thaliana.

Importantly, a synthetic peptide of 24 amino acids (aa) (called nlp24) derived from HaNLP3 of $H$. arabidopsidis induces defense responses in A. thaliana, e.g., ethylene production, and immunity to $H$. arabidopsidis (Oome et al. 2014). In parallel, a synthetic peptide of 20 aa (called as nlp20) derived from PpNLP of $P$. parasitica induces defense responses of Arabidopsis, including ethylene production (Böhm et al. 2014). The nlp24 peptide of HaNLP3 is strongly conserved in both cytotoxic and noncytotoxic type 1 NLPs, further confirming that NLPs commonly possess a MAMP signature recognized by Arabidopsis (Oome et al. 2014). The sensitivity to nlp20 is detected in some of the Brassicaceae species and Lactuca sativa (lettuce) (Böhm et al. 2014). Recently, RLP23, the leucine-rich repeat receptor protein, was identified as the receptor of the nlp20 peptide in A. thaliana (Albert et al. 2015), revealing that recognition of the nlp MAMP by A. thaliana depends on a typical pattern recognition receptor.

Consistent with the finding that $H$. arabidopsidis possesses noncytotoxic NLPs, 11 of 19 NLPs derived from oomycete pathogen Phytophthora sojae were shown to lack cytotoxic activities (Dong et al. 2012). P. sojae represents a hemibiotrophic lifestyle, i.e., initial biotrophic infection subsequently switching to necrotrophy. Importantly, cytotoxic $N L P$ genes are expressed at 
the onset of necrotrophic growth, whereas noncytotoxic NLP genes are expressed preferentially during biotrophic infection, suggesting functional diversification among the NLPs of $P$. sojae (Dong et al. 2012; Qutob et al. 2002). This tendency is clear in NLPs of ascomycete fungal pathogen Colletotrichum higginsianum, which represents a hemibiotrophic lifestyle (Kleemann et al. 2012; O'Connell et al. 2012; Perfect et al. 1999).

The ascomycete Colletotrichum species infect a wide range of plant species including many important crops, although each species generally exhibits a narrow and specific host range. Colletotrichum fungi develop specialized infection structures

A

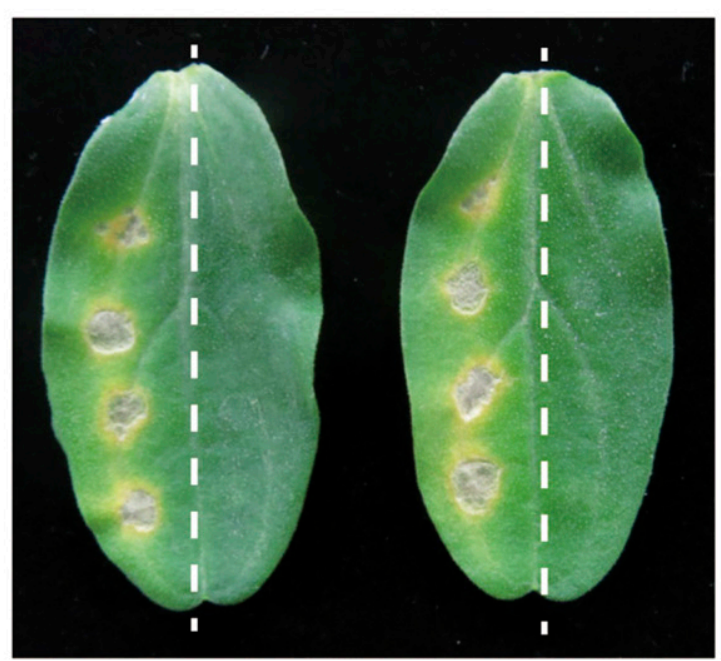

WT NLP1 CE-1

WT NLP1 CE-2

C
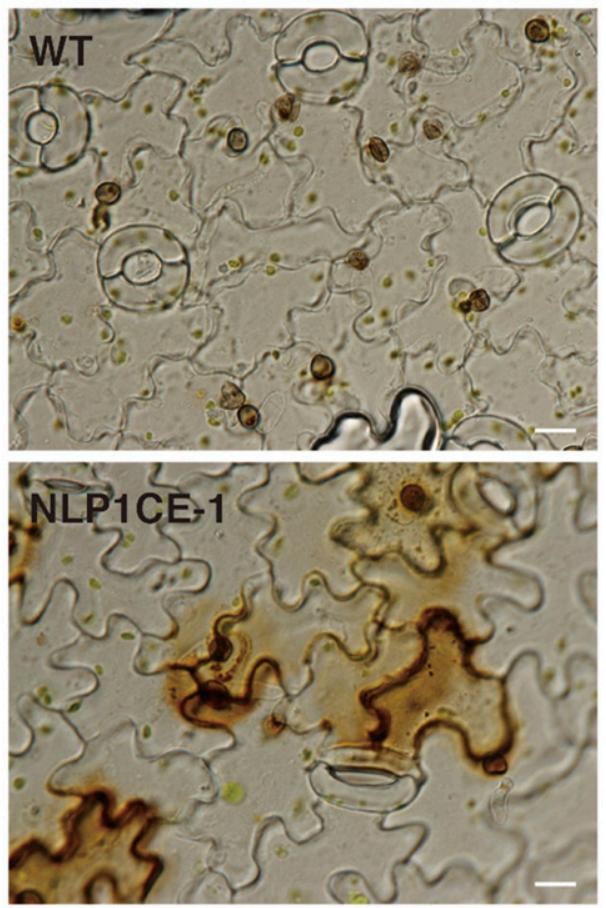

called appressoria that are darkly pigmented with melanin (Kubo and Takano 2013). The genome sequence analyses of Colletotrichum orbiculare, which causes cucumber anthracnose disease, uncovered the presence of multiple NLPs in the pathogen (Gan et al. 2013). Among them, a cytotoxic-type NLP named NLP1 is preferentially expressed at late biotrophic and necrotrophic phases (Irieda et al. 2014), consistent with the case of $C$. higginsianum (Kleemann et al. 2012).

The restricted expression of cytotoxic NLPs in the late infection phase in multiple fungal pathogens raises the possibility that the expression of cytotoxic NLPs must be suppressed at an
B

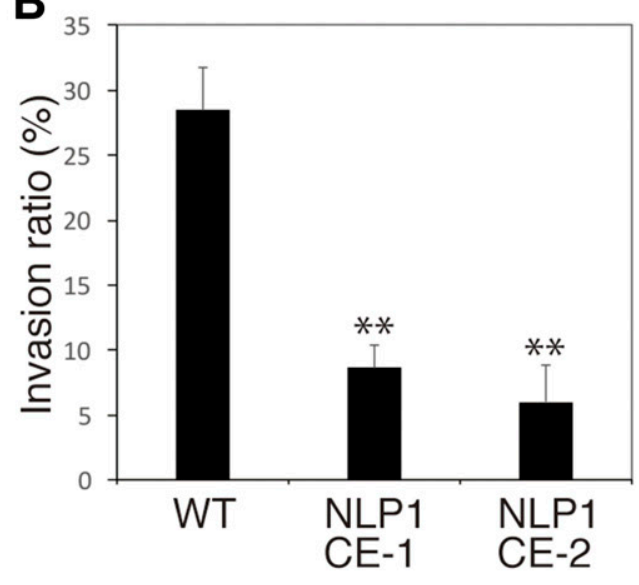

D

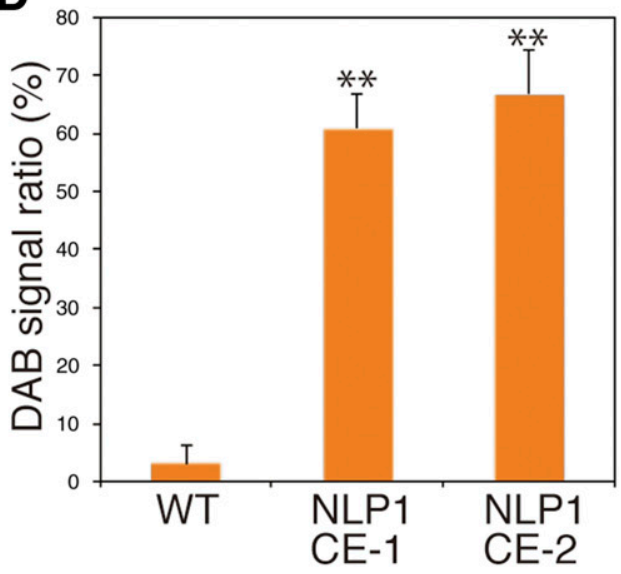

$\mathbf{E}$

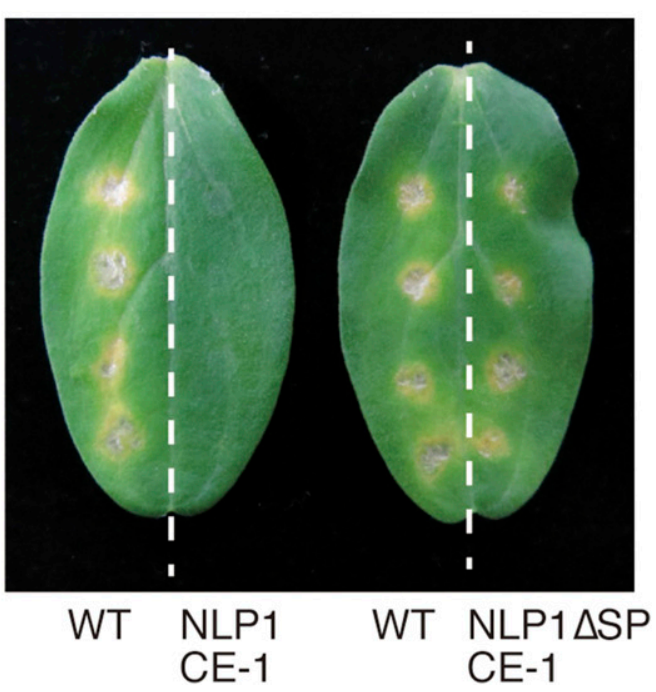


early infection stage for successful infection of each pathogen; in other words, the inappropriate expression of cytotoxic NLPs might have negative impacts on the full virulence of the pathogens. To assess this possibility, in this study, we generated C. orbiculare transgenic strains constitutively expressing the cytotoxic protein NLP1. The generated strains were subsequently inoculated on the host plant cucumber to investigate whether the inappropriate expression of cytotoxic NLP1 interferes with $C$. orbiculare host infection. We found that the constitutive expression of NLP1 in $C$. orbiculare completely blocked pathogen infection on cucumber, supporting our hypothesis that the expression of cytotoxic NLP must be regulated properly for successful infection of the pathogen. However, we found that the NLP1-dependent virulence suppression is uncoupled from its cytotoxic activity. Surprisingly, further studies revealed that the $\mathrm{C}$-terminal region but not the region containing nlp24 of the NLP1 protein is recognized by Cucurbitaceae plants and that this recognition results in strong defense activation that can terminate $C$. orbiculare infection.

\section{RESULTS}

\section{Constitutive expression of NLP1 in C. orbiculare enhanced cucumber defense and impaired fungal virulence on cucumber.}

To generate the $C$. orbiculare transgenic strains constitutively expressing CoNLP1 (hereafter called NLP1) under the translation elongation factor gene $(T E F)$ promoter of Aureobasidium pullulans (Vanden Wymelenberg et al. 1997), we transformed the $C$. orbiculare wild-type strain 104-T (WT) with a plasmid pBATTEFPNLP1 (details below). We inoculated the obtained transformants of $C$. orbiculare on host plant cucumber (Cucumis sativus) to assess possible effects of the constitutive expression of NLP1 on fungal virulence (Fig. 1A). Remarkably, the inoculation assay revealed that six of 12 transformants did not develop any lesions on cucumber, whereas the WT strain caused severe lesions, indicating that the constitutive expression of NLP1 under the control of the TEF promoter impaired fungal virulence of $C$. orbiculare on host cucumber (Fig. 1A). The transgenic lines with a failure of infection, named NLP1CE strains, grew normally on nutrient-rich media similar to the nontransgenic WT strain (Supplementary Fig. S1). Microscopic analysis showed that the NLP1CE strains failed to develop invasive hyphae effectively, compared with the WT strain (Fig. 1B). The invasion ratio of the NLP1CE strain was less than $10 \%$, whereas that of the WT strain was approximately $28 \%$ (Fig. 1B). We also found that the length of the invasive hyphae produced by appressoria of the NLP1CE strains was shorter than those produced by appressoria of the WT strain (Supplementary Fig. S2).

These findings raised the possibility that the route of entry for the NLP1CE strains was blocked via enhanced activation of preinvasive defense in cucumber. To assess this, reactive oxygen species (ROS) generation was investigated, using 3,3diaminobenzidine (DAB) staining, and it was found that strong DAB signal was detected around the inoculation sites of the NLP1CE strains in cucumber whereas the signal was undetectable in the WT strain (Fig. 1C and D). This finding indicates that the NLP1CE strains elicited ROS generation in cucumber. The assay for papillary callose deposition also suggested that appressoria of NLP1CE strains strongly induced callose deposition in cucumber as compared with WT (Supplementary Fig. S3). These results suggest that the NLP1CE strains of $C$. orbiculare triggered substantial preinvasive immune responses of cucumber in comparison with the WT strain.

Because NLP1 is a secreted protein, we next asked whether the secretion of NLP1 is critical for NLP1-dependent reduced virulence on cucumber. To assess this point, we generated the $C$. orbiculare strains expressing NLP1 lacking its signal peptide (NLP1 $\triangle \mathrm{SP})$ under the control of the TEF promoter. The inoculation assay of the strains expressing NLP1 $\triangle$ SP (NLP1 $\triangle$ SPCE strains) on cucumber revealed that the NLP1 $\triangle$ SPCE strains retained the WT-level virulence on cucumber (Fig. 1E). This supports the idea that NLP1 needs to be secreted from the pathogen to trigger preinvasive immune responses of cucumber.

\section{Multiple plant species are resistant \\ to $C$. orbiculare constitutively expressing NLP1.}

Next, we asked whether NLP1 expression by $C$. orbiculare also affects the pathogen virulence to other susceptible plants in addition to cucumber. We first performed the inoculation assay of the NLP1CE strains in three additional Cucurbitaceae cultivars, melon (Cucumis melo), winter melon (Benincasa hispida), and long melon (Cucumis melo L. var utilissimus) (Fig. 2A). As a result, we found that the NLP1CE strains failed to infect all tested Cucurbitaceae cultivars whereas the WT strain developed severe lesions in these cultivars (Fig. 2A). The results indicate that constitutive expression of NLP1 by $C$. orbiculare enhances the resistance of multiple Cucurbitaceae cultivars toward the pathogen. It is known that C. orbiculare, including strain 104-T used in this study, infects Nicotiana benthamiana, belonging to the family Solanaceae

Fig. 1. Constitutive expression of NLP1 in Colletotrichum orbiculare impaired pathogen infection on cucumber via defense activation. A, Inoculation assay of the $C$. orbiculare strains constitutively expressing NLP1 on host cucumber. The wild-type (WT) strain 104-T was inoculated onto the left halves of the cucumber cotyledons as a positive control. The test strains were inoculated on the right halves. Inoculated leaves were incubated for 7 days. Among the 12 transformants tested, six transformants failed to develop lesions on cucumber and the results of two transformants (NLP1CE-1 and NLP1CE-2) are shown here. Similar results were obtained from three independent experiments. B, Quantitative assay for appressorium-mediated invasion on cucumber. A conidial suspension of each strain was inoculated onto the abaxial surface of cucumber cotyledons and inoculated plants were incubated for 4 days. The ratio of appressoria forming invasive hyphae was calculated. In each experiment, at least 150 appressoria were examined and counted to calculate the percentage of invasive hyphae. Means and standard deviations were calculated from three independent experiments. The statistical significance of differences between means was determined by two-tailed $t$ test. Two asterisks (**) indicate $P<0.01$ (comparison with wild-type). C, 3,3'-diaminobenzidine (DAB) staining assay for the detection of $\mathrm{H}_{2} \mathrm{O}_{2}$ accumulation. A conidial suspension of each strain was inoculated onto the abaxial surface of cucumber cotyledons and leaves were incubated for 2 days. Cotyledons were then stained with DAB and were subjected to microscopic observation (note: appressoria do not develop invasive hyphae at this time, even in the WT strain). Dark staining indicates $\mathrm{H}_{2} \mathrm{O}_{2}$ accumulation. Similar results were obtained from three independent experiments. Bars = $10 \mu \mathrm{m}$. D, Quantitative analysis for DAB staining. Mean percentage of $\mathrm{H}_{2} \mathrm{O}_{2}$ accumulation at sites of attempted penetration in each strain. DAB-staining ratio was calculated as the mean percentage of appressoria with the penetration pore that displayed dark-stained area per total appressoria with the penetration pore. In each experiment, approximately 150 appressoria with the penetration pore were counted for each strain. Means and standard deviations were calculated from three independent experiments. The statistical significance of differences between means was determined by two-tailed $t$ test. Two asterisks ( $* *$ ) indicate $P<$ 0.01 (comparison with wild-type). E, Inoculation assay of the strains expressing NLP1 lacking the signal peptide (NLP1 $\triangle$ SPCE-1). The WT strain 104-T was inoculated onto the left halves as a positive control. On the right halves of the cucumber cotyledons, the test strains were inoculated. Inoculated plants were incubated for 7 days. Similar results were obtained from three independent experiments.
} 
(Shen et al. 2001; Takano et al. 2006). Thus, we investigated the effects of NLP1 expression on the virulence of $C$. orbiculare toward $N$. benthamiana via the inoculation assay. In contrast to cucumber, the NLP1CE strains clearly developed lesions in $N$. benthamiana, indicating that the NLP1CE strains retained virulence on $N$. benthamiana (Fig. 2B). However, the size of the lesions developed by NLP1CE strains was relatively smaller than the size of lesions developed by the WT strain (Fig. 2B and C). Thus, the constitutive expression of NLP1 in C. orbiculare likely enhanced the resistance of $N$. benthamiana to some degree but was not sufficient to block the infection by $C$. orbiculare.

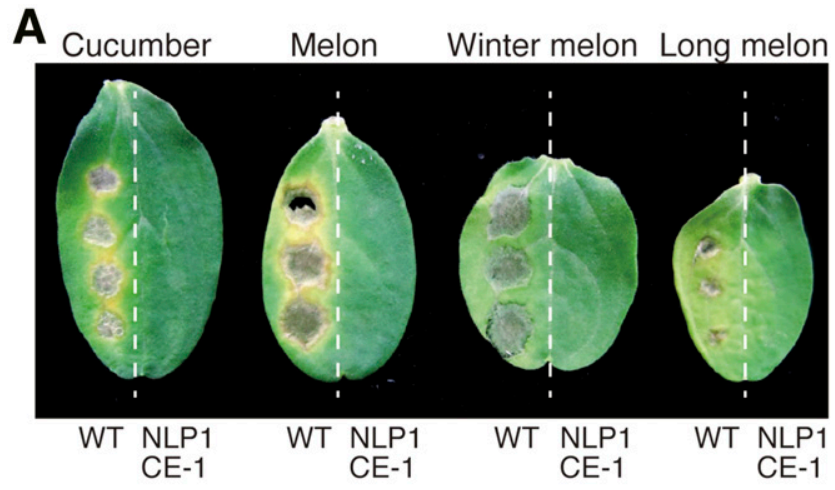

B
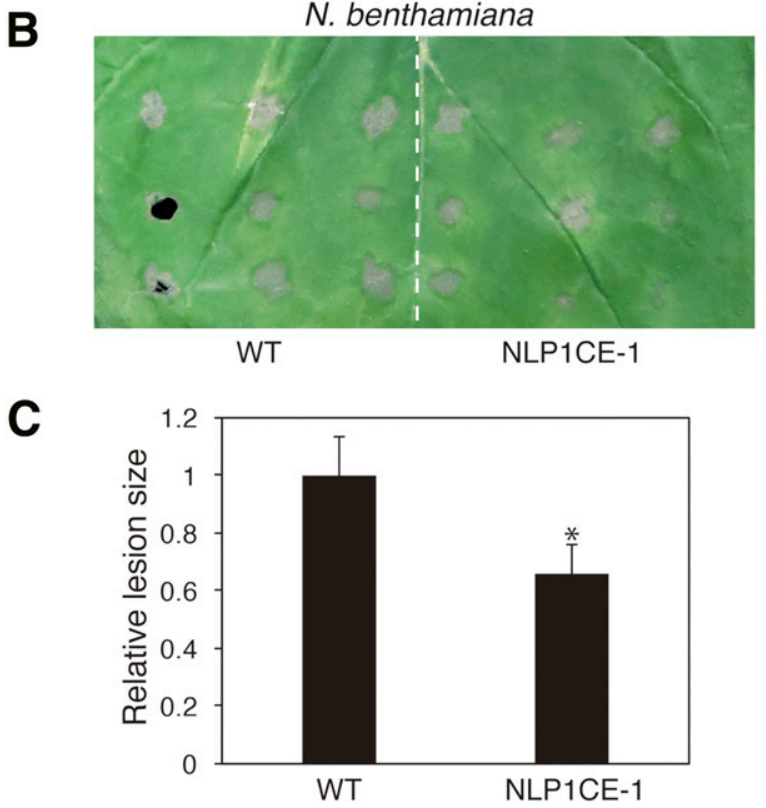

Fig. 2. Constitutive expression of NLP1 completely blocked the pathogen infection in multiple Cucurbitaceae cultivars but not in Nicotianaa benthamiana. A, Inoculation assay of the strain NLP1CE-1 on multiple Cucurbitaceae cultivars. The wild-type strain 104-T (WT) was inoculated onto the left halves of the cotyledons derived from each cultivar. NLP1CE-1 was inoculated onto the right halves of the cotyledons. Inoculated leaves were incubated for 7 days. Similar results were obtained from three independent experiments. B, Inoculation assay of the strain NLP1CE-1 on $N$. benthamiana. The WT strain 104-T was inoculated on the left half of a $N$. benthamiana leaf. The test strain was inoculated on the right half. Compared with WT, NLP1CE-1 delayed lesion development with a slight reduction of lesion size. Photographs were taken at 7 days postinoculation (dpi). Similar results were obtained from five independent experiments. C, Quantitative assay for lesion development area in N. benthamiana inoculated with 104-T (WT) and NLP1CE-1. The inoculated leaves were incubated for 7 days. For each strain, the total lesion area for 36 inoculated spots was quantified with Image $\mathbf{J}$ software. Three independent replicate experiments were performed with similar results. The statistical significance of differences between means was determined by two-tailed $t$ test. An asterisk $(*)$ indicates $P<0.05$ (comparison with wild-type).

\section{Cytotoxic activity is not related}

to NLP1-mediated enhanced defense.

As mentioned earlier, it is known that multiple NLPs of other fungi have cytotoxic activity toward $N$. benthamiana. Therefore, we investigated whether NLP1 of $C$. orbiculare also induces cell death in $N$. benthamiana, by Agrobacterium-mediated transient expression of NLP1. The transient expression of NLP1 resulted in lesion development in $N$. benthamiana, showing a cytotoxic activity leading to cell death in the plant (Fig. 3A). We next asked whether this activity is related to the negative effect of constitutive NLP1 expression on $C$. orbiculare virulence. Based on

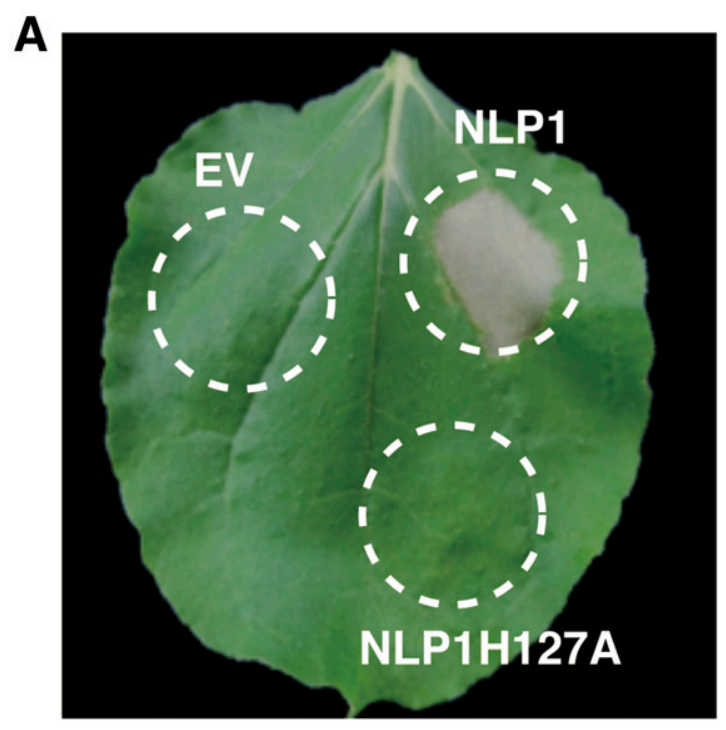

B

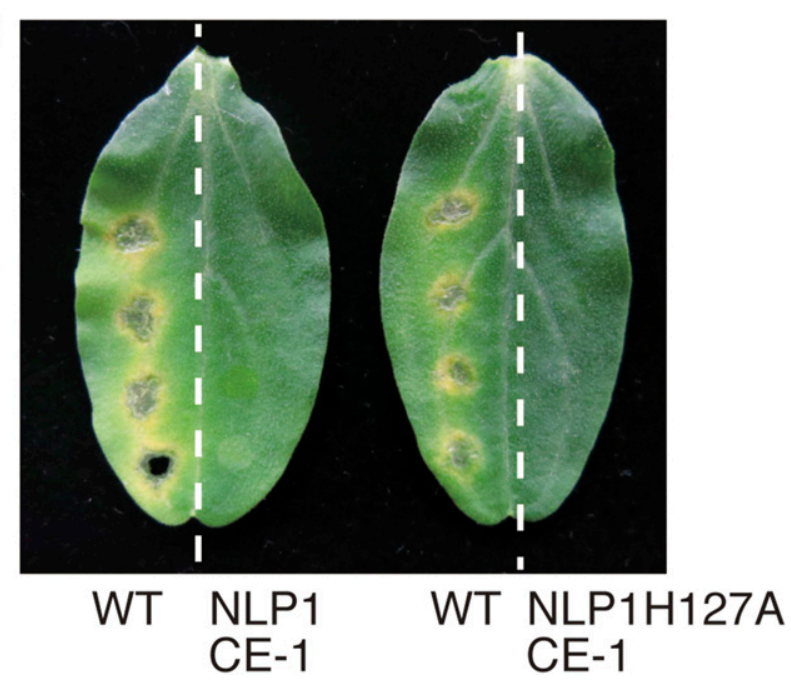

Fig. 3. NLP1-dependent reduced virulence is uncoupled from its cytotoxic activity. A, Agrobacterium-mediated transient expression of NLP1 and NLP1 lacking the cytotoxic activity in Nicotiana benthamiana leaves. Agrobacterium tumefaciens carrying empty vector pBICP35 (EV), pBICP35 carrying full-length $N L P 1$ (NLP1), and pBICP35 carrying $N L P 1$ having a mutation for cytotoxic activity (NLP1H127A) were infiltrated into $N$. benthamiana leaves. The infiltration sites were represented by dashed circles. The cell death symptoms were assessed at 5 days after the infiltration. Similar results were obtained from three independent experiments. B, Inoculation of the Colletotrichum orbiculare strain expressing NLP1H127A (NLP1H127ACE-1) on cucumber. The wild-type strain 104-T (WT) was inoculated onto the left halves of cotyledons as a positive control. The test strains were inoculated onto the right halves. The inoculated cotyledons were incubated for 7 days. Similar results were obtained from three independent experiments. 
Ottmann et al. (2009), we generated a mutated NLP1 carrying a mutation changing histidine 127 to alanine that is expected to lose its cytotoxic activity; we confirmed that the transient expression of this mutant NLP1 (named NLP1H127A) did not cause lesion development in $N$. benthamiana, showing that NLP1H127A lost its cytotoxic activity toward the plant (Fig. $3 \mathrm{~A}$ ). We then generated the $C$. orbiculare strain constitutively expressing NLP1H127A (named NLP1H127ACE) and inoculated the strain on cucumber. The NLP1H127ACE strain failed to infect cucumber, which is indistinguishable from the phenotype of the NLP1CE strain (Fig. 3B). This result indicates that the constitutive expression of NLP1H127A, as well as NLP1, impairs fungal virulence of $C$. orbiculare on cucumber and, therefore, the cytotoxic activity of NLP1 is unlikely to be coupled with the NLP1dependent reduction of $C$. orbiculare virulence toward cucumber. Compared with the WT strain, the NLP1H127ACE strain also showed slightly reduced virulence on $N$. benthamiana, similar to that of the NLP1CE strain (Supplementary Fig. S4A).

\section{The nlp24 MAMP sequence recognized by $A$. thaliana is not related to the enhanced defense in cucumber.}

It has been recently reported that a peptide sequence relatively conserved inside NLP proteins is recognized as a MAMP by A. thaliana (Böhm et al. 2014; Oome et al. 2014). Based on the finding that the expression of NLP1H127A lacking the cytotoxic activity can reduce the virulence of $C$. orbiculare, the same as the expression of the intact NLP1, we next investigated the relationship of the MAMP sequence of NLP1 to the negative impact on virulence. It has been reported that the sequence AIMYAWYFPKDSPMLLMGHRHDWE (Hanlp24) in HaNLP3 of $H$. arabidopsidis is recognized by $A$. thaliana and solely elicits the defense responses of $A$. thaliana (Fig. 4A) (Oome et al. 2014). It was also reported that the corresponding region in PpNLP of $P$. parasitica (AIMYSWYFPKDSPVTGLGHRHDWE) is recognized by $A$. thaliana accompanied with activation of the defense responses (Böhm et al. 2014). C. orbiculare NLP1 also has a typical MAMP sequence (AIMYSWYMPKDSPSTGLGHRHEWE, named Conlp24), and we found that the synthetic peptide for Conlp24, indeed, elicits ROS generation in A. thaliana (Fig. 4A and $\mathrm{B}$ ), similar to the previous report on ROS generation by the nlp20 peptides of PpNLP in A. thaliana (Albert et al. 2015; Böhm et al. 2014). We then generated a mutant NLP1 lacking the nlp24 MAMP sequence. Based on the reported mutational analyses to nlp20 of PpNLP (Böhm et al. 2014), we decided to substitute isoleucine (position 2), methionine (position 3), and tyrosine (position 4) in Conlp24 with alanine, designated as Conlp24Mut, and confirmed that the synthetic peptide for Conlp24Mut failed to elicit ROS generation in A. thaliana (Fig. 4A and B). We then generated $C$. orbiculare strains expressing the mutated NLP1 having the corresponding mutations (named NLP1Mut) and the generated $C$. orbiculare strains designated NLP1MutCE were subjected to inoculation assay on cucumber. The $C$. orbiculare NLP1MutCE strains were still not able to infect cucumber as with the NLP1CE strains (Fig. 4C). Collectively, the findings suggest that the negative effect of NLP1 expression on $C$. orbiculare virulence is uncoupled from the reported nlp20/24 MAMP sequence and its cytotoxic activity in NLP1. The NLP1MutCE strain also had slightly reduced virulence on $N$. benthamiana, the same as the NLP1CE strain.

\section{The carboxyl-terminal region of NLP1}

is recognized by Cucurbitaceae cultivars.

The findings raised the possibility that secreted NLP1 is recognized by cucumber via a region distinct from Conlp24 sensed by $A$. thaliana. To determine the responsible region, we decided to generate a deletion series of NLP1 that are sequentially deleted from its carboxy terminus, i.e., the deletion
A
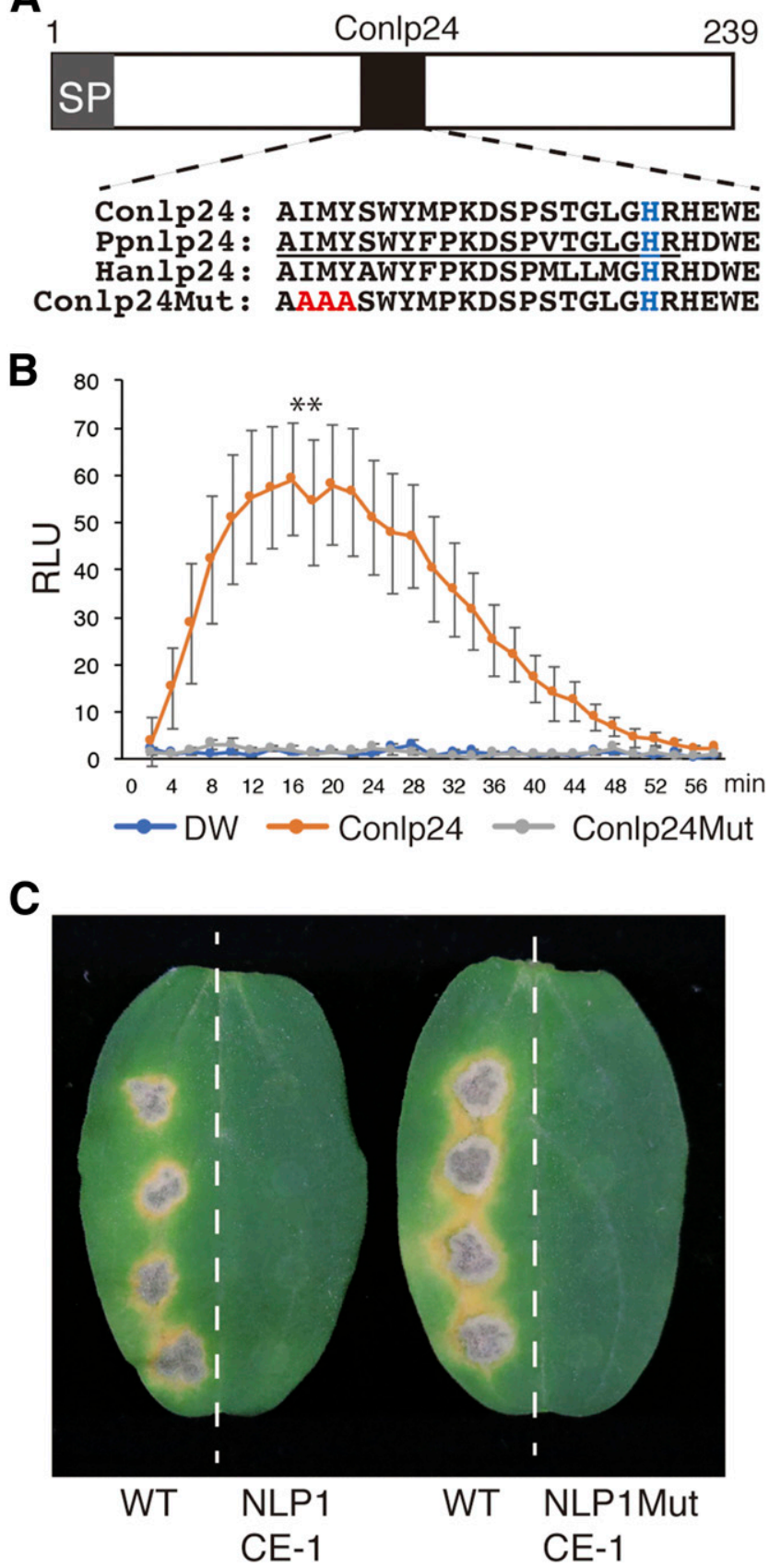

Fig. 4. The nlp24 sequence of NLP1 is not essential to trigger enhanced defense in cucumber. A, Schematic representation of nlp24 in Colletotrichum orbiculare NLP1 with introduced alanine substitutions. nlp24 of $C$. orbiculare (Conlp24) is aligned with nlp24 of Phytophthora parasitica (Ppnlp24) and of Hyaloperonospora arabidopsidis (Hanlp24). Conlp24 with alanine substitutions was also aligned (Conlp24Mut). The Ppnlp20 sequence is underlined. The closed box represents the Conlp24 region. SP represents the signal peptide of NLP1. B, Reactive oxygen species production induced by $1 \mu \mathrm{M}$ Conlp24, $1 \mu \mathrm{M}$ Conlp24Mut, or distilled water (DW) was monitored, using a luminol-based assay in leaf discs of Arabidopsis Col-0. Data are given as relative light units (RLU) and represent the mean \pm standard error of 12 wells from one representative experiment of three. The statistical significance of differences between means of total RLU was determined by two-tailed $t$ test. Two asterisks (**) indicate $P<0.01$ (comparison with DW treatment). C, Inoculation assay of the strain expressing the mutated NLP1 carrying Conlp24Mut (NLP1MutCE-1) on host cucumber. The wild-type strain 104-T (WT) was inoculated onto the left halves of cucumber cotyledons as a positive control. The test strains were inoculated onto the right halves. The inoculated plants were incubated for 7 days. Similar results were obtained from three independent experiments. 
of 22, 42, and 62 aa (Note: all deleted NLP1 forms still retain the reported nlp24 MAMP sequence and the domain for cytotoxic activity) (Fig. 5A).

We then generated $C$. orbiculare transgenic lines expressing these truncated forms of NLP1 under the control of the TEF promoter (Fig. 5A). We found that the expression of all NLP1 $\Delta 22$, NLP1 $\Delta 42$, and NLP1 $\Delta 62$ proteins had no effects on the virulence of $C$. orbiculare, whereas the expression of full-length NLP1 completely blocked the infection of $C$. orbiculare on cucumber (Fig. 5B). The result clearly indicates that the deletion of the 22aa carboxyl terminal region abolishes the NLP1 effects on C. orbiculare virulence to cucumber and, also, raises the possibility that the corresponding carboxyl-terminal region of NLP is recognized by cucumber, which leads to the activation of the cucumber defense against the pathogen.

To assess this possibility, we investigated whether the expression of the carboxyl-terminal region in C. orbiculare is sufficient for cucumber defense activation. We generated $C$. orbiculare lines that secrete a red fluorescent protein, mCherry, fused to the C-terminus 32 aa of NLP1 named mCNLP1C32 (Fig. 6A), because we considered that the addition of mCherry can mimic the possible recognition of the C-terminal region of NLP1 compared with the sole expression of the $\mathrm{C}$-terminal region as a short peptide. We first constructed a fungal transformation vector carrying the signal peptide derived from the effector DN3 (SP): mCherry:NLP1 $\triangle \mathrm{SP}$, called mCNLP1FL, as a control, and introduced it into $C$. orbiculare (Fig. 6A). The obtained mCNLP1FL transformants were subjected to inoculation assay on cucumber. Whereas all transformants expressing SP:mCherry infected both cucumber and $N$. benthamiana the same as the wild-type strain (Supplementary Fig. S5), of 16 tested mCNLP1FL transformants (SP:mCherry:NLP1 $\triangle \mathrm{SP}$ ), four transformants clearly exhibited reduced virulence on cucumber (Fig. 6B). This suggests that the

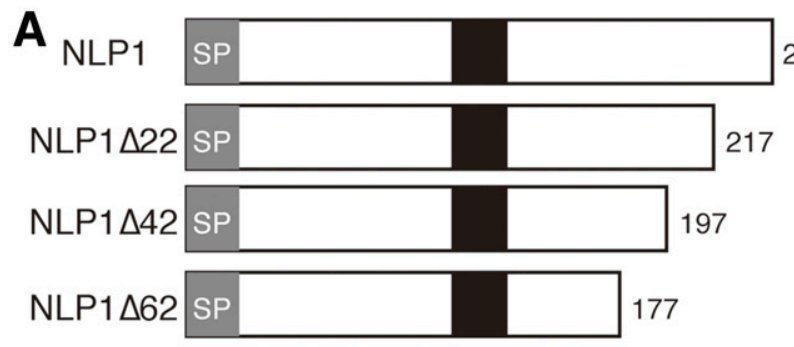

B

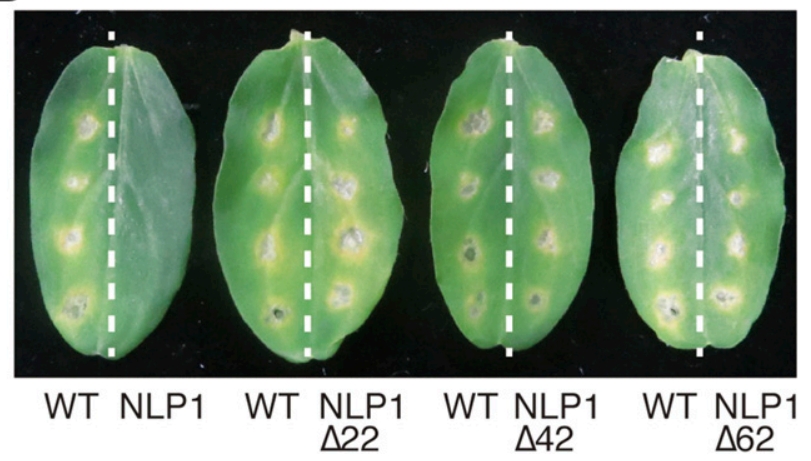

Fig. 5. The C-terminal region of NLP1 is essential for triggering cucumber defense. A, Schematic representation of the truncated NLP1 series deleted from its $\mathrm{C}$ terminus. The closed box represents the Conlp24 region. SP represents the signal peptide of NLP1. B, Inoculation assay of the Colletotrichum orbiculare strains expressing the deleted versions of NLP1. The wild-type strain 104-T (WT) was inoculated onto the left halves of cucumber. On the right halves, the test strains were inoculated. The inoculated cotyledons were incubated for 7 days. Similar results were obtained from three independent experiments. secreted mCherry:NLP1 $\triangle$ SP activates cucumber defense via its recognition, similar to NLP1 without mCherry. We then constructed a fungal transformation vector carrying SP:mCherry: NLP1C32 (Fig. 6A) and introduced it into C. orbiculare. Of 48 mCNLP1C32 transformants, three failed to infect cucumbers, in contrast to the WT strain (Fig. 6B), which is similar to C. orbiculare strains expressing NLP1 or SP:mCherry:NLP1 $\triangle \mathrm{SP}$.

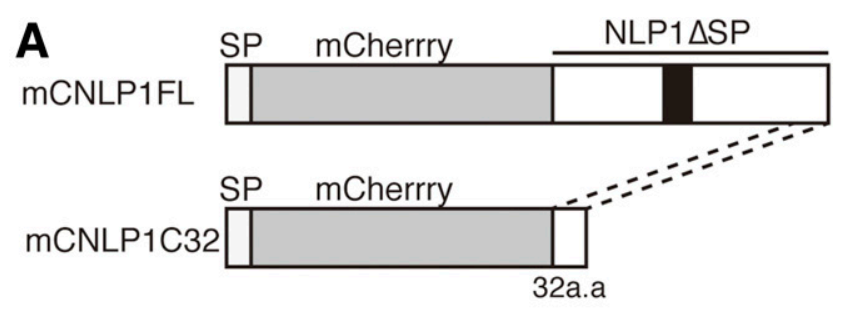

B

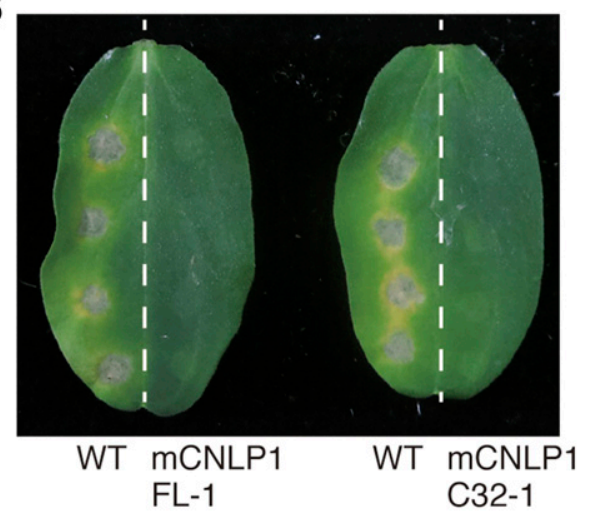

C

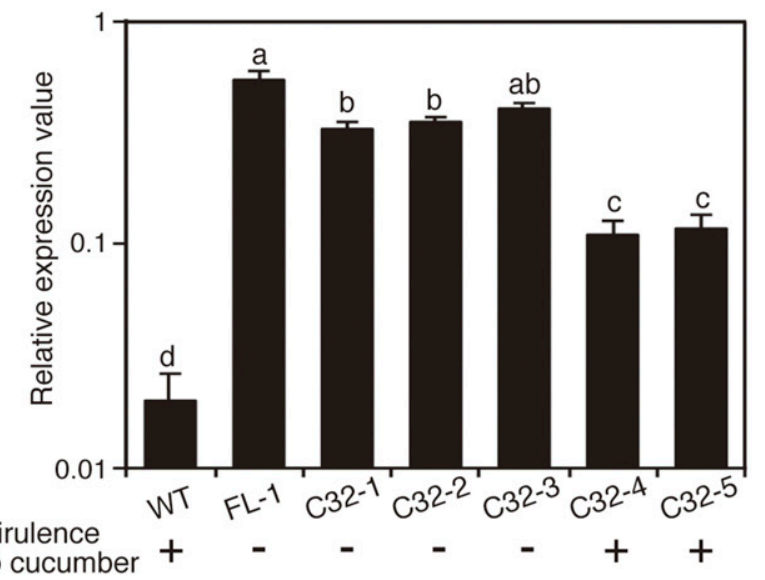

Fig. 6. Expression of the C-terminal 32 amino acids (aa) of NLP1 as a fusion with SP:mCherry is sufficient to trigger cucumber defense. A, Schematic diagram for SP:mCherry fused to the NLP1 lacking its signal peptide (SP) (mCNLP1FL) or fused to the C-terminal 32 aa of NLP1 (mCNLP1C32). The closed box represents the Conlp24 region. B, Inoculation assay of the strain expressing SP:mCherry fused to the C-terminal 32 aa of NLP1. The wild-type strain 104-T (WT) was inoculated onto the left halves of cucumber cotyledons as a positive control. Test strains (mCNLP1FL-1 and mCNLP1C32-1) were inoculated on the right halves. The inoculated leaves were incubated for 7 days. Similar results were obtained from three independent experiments. C, Quantitative reverse transcription-polymerase chain reaction analysis for expression of the gene encoding SP:mCherry fused to the C-terminal 32 aa of NLP1. Conidial suspensions of tested strains were inoculated on cucumber cotyledons and were incubated for 1 day. Means and standard deviations were calculated from three independent experiments. The statistical significance of differences between means was determined on log-transformed data by Tukey test. Means not sharing the same letter are significantly different $(P<0.05)$. FL1 = mCNLP1FL-1; C32-1 to C32-5 = mCNLP1C32-1 to mCNLP1C32-5; $+=$ WT-like virulence to cucumber; $-=$ no virulence. 
Thus, the finding supports the idea that the 32-aa carboxylterminal region of NLP1 (NLP1C32) is recognized by cucumber.

We further assessed why the other 45 of the 48 mCNLP1C32 transformants tested retained virulence on cucumber. We hypothesized that the transformants with full virulence might exhibit lower expression of NLP1C32 compared with the transformants lacking virulence. Indeed, quantitative reverse transcription-polymerase chain reaction (qRT-PCR) analysis of the SP:mCherry:NLP1C32 gene during the preinvasive infection phase of $C$. orbiculare showed that the expression levels in the three transformants lacking virulence (mCNLP1C32-1 to mCNLP1C32-3) in this infection phase were several-fold higher than that in the transformants with full virulence (mCNLP1C32-4 and mCNLP1C32-5) (Fig. 6C). The finding strongly suggests that i) NLP1C32 is recognized by cucumber and ii) the threshold of the expression level of NLP1C32 is likely to be present to elicit the cucumber defense.

Next, melon and $N$. benthamiana were inoculated with the mCNLP1C32 strains that failed to infect cucumber (Fig. 7). Importantly, the mCNLP1C32 strains completely lacked virulence on melon but retained virulence on $N$. benthamiana with a slight reduction (Fig. 7), which was identical to the mCNLP1FL strains and NLP1CE strains that secrete the fulllength NLP1. In contrast, we found that a mutant defective in the CST1 (Colletotrichum Ste12-like) gene lacked virulence on both melon and N. benthamiana (Fig. 7), because the CST1 gene is required for a fundamental process of $C$. orbiculare plant infection (Tsuji et al. 2003). Therefore, the NLP1C32 expression in $C$. orbiculare mimics the unique virulence-related phenotype of $C$. orbiculare constitutively expressing NLP1. Collectively, these findings indicate that the $\mathrm{C}$-terminal region of NLP1 is recognized by Cucurbitaceae cultivars and, then, strongly activates their defense to terminate $C$. orbiculare infection. The NLP1C32 sequence is highly conserved in NLP1 homologs of other fungi and oomycetes (Supplementary Fig. S6), implying possible plant recognition of these NLPs at their C-terminal region.

\section{DISCUSSION}

In this report, we have revealed that the constitutive expression of NLP1 in C. orbiculare strongly reduced the virulence of the pathogen toward its host plant cucumber by inducing cucumber defense. Cytotoxic NLPs are generally expressed at the late infection phase, such as during the switching phase from biotrophy to necrotrophy (Dong et al. 2012; Qutob et al. 2002). Thus, these findings suggest that the tight regulation of NLP1 expression is important and the inappropriate expression of NLP1 has a negative impact on virulence. In contrast with our results, it was reported that overexpression of a Fusarium NLP1 (NEP1) in Colletotrichum coccodes resulted in enhanced virulence on Abutilon theophrasti and expanded its host range to tomato and

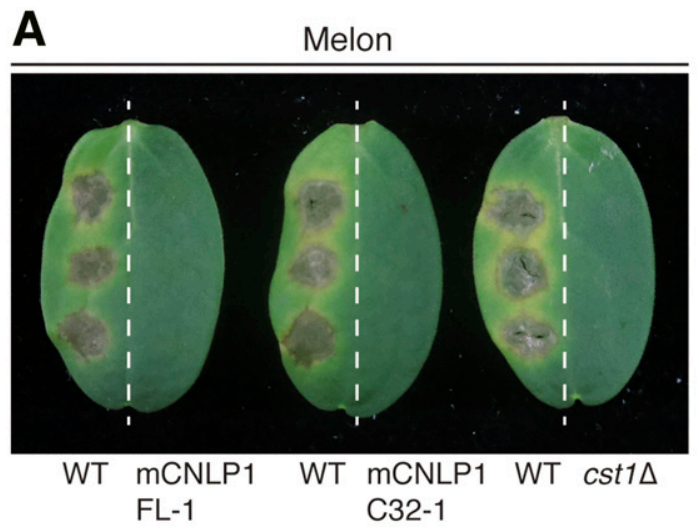

B

N. benthamiana

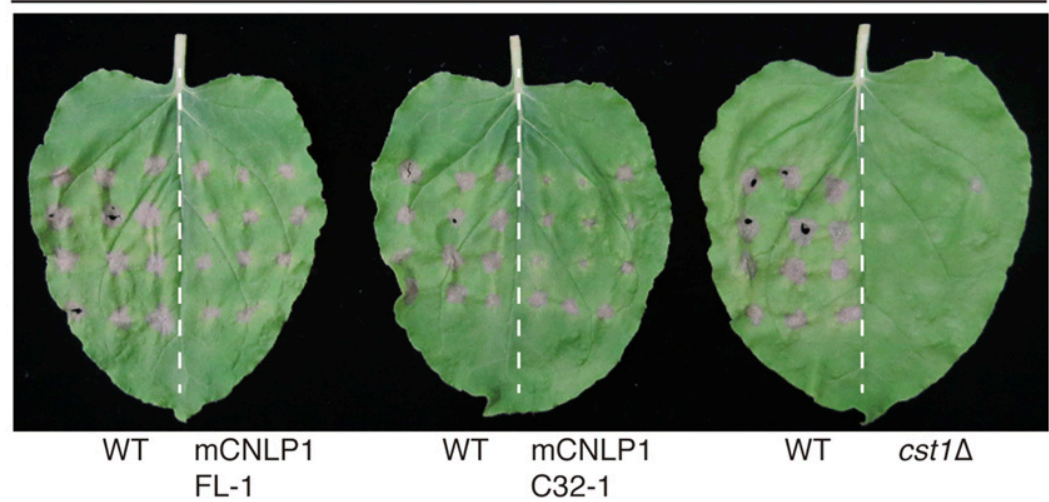

Fig. 7. The virulence phenotype of strain mCNLP1C32-1 is identical to that of strain mCNLP1FL-1 but distinct from that of the $c$ st 1 pathogenicity mutant. A, Inoculation assay on melon. Colletotrichum orbiculare 104-T (WT) was inoculated on the left halves of cotyledons. The test strains were inoculated on the right halves. The inoculated cotyledons were incubated for 7 days. The strain mCNLP1FL-1 expresses SP:mCherry fused to the NLP1 lacking its signal peptide, whereas mCNLP1C32-1 expresses SP:mCherry fused to the C-terminal 32 amino acids (aa) of NLP1. The cst 1 null mutant (cst1 $\Delta$ ) is defective in pathogenicity because CST1 is a transcription factor essential for pathogenicity. Similar results were obtained from three independent experiments. B, Inoculation assay on Nicotiana benthamiana leaves. Inoculated leaves were incubated for 7 days. The WT developed severe lesions in $N$. benthamiana. Notably, the mCNLP1FL-1 and mCNLP1C32-1 strains developed lesions in N. benthamiana, although the virulence of these strains was reduced compared with WT. In contrast, the cst1 $\Delta$ mutant failed to develop them. Thus, the C-terminal 32 aa of NLP1 is sufficient to mimic the unique effect of full-length NLP expression in $C$. orbiculare infection on cucumber. Similar results were obtained from three independent experiments. 
tobacco (Amsellem et al. 2002). Importantly, we found that NLP1-mediated reduced virulence does not depend on its cytotoxic activity, via characterization of $C$. orbiculare strains expressing NLP1 lacking the cytotoxic activity (Fig. 3).

We then asked whether the NLP1-mediated reduced virulence is related to the putative nlp24/nlp20 that can be recognized by A. thaliana (Böhm et al. 2014; Oome et al. 2014). We found that the synthetic peptide corresponding to the nlp24 region of $C$. orbiculare NLP1 can activate the defense response of $A$. thaliana (Fig. 4B), confirming that NLP1 contains an active nlp24 MAMP signature. We generated $C$. orbiculare expressing a mutant NLP1 lacking functional nlp24; analyses on these strains indicated that the reduced virulence mediated by NLP1 was uncoupled from the nlp24 of NLP1 (Fig. 4C). It has been reported that the nlp20 of PpNLP in $P$. parasitica is recognized as a MAMP sequence by some Brassicaceae species and Lactuca sativa but not by other plants such as N. benthamiana, Triticum aestivum, and Solanum lycopersicum (Böhm et al. 2014). Our data suggests that the nlp24/nlp20 sequence is also unlikely to be recognized by cucumber.

Through the deletion analyses of NLP1 combined with the mCherry fusion assay, we found that the carboxy-terminal 32 aa of NLP1 is able to elicit cucumber defense via its recognition (Fig. 6). Importantly, this recognition system is conserved in multiple other Cucurbitaceae plants (Figs. 2A and 7A). Expression of NLP1 in $C$. orbiculare also resulted in a slight reduction of virulence on $N$. benthamiana (Figs. $2 \mathrm{~B}$ and $\mathrm{C}$ and 7B). The inoculation assay of $C$. orbiculare expressing the mutated NLPs (H127A and Conlp24Mut) suggested that the reduced virulence mediated by NLP1 on N. benthamiana is uncoupled from its cytotoxic activity and the nlp24 sequence, i.e., $N$. benthamiana is likely to recognize the carboxyl terminal region of NLP1, however, the plants fail to mount an effective defense against $C$. orbiculare for an unknown reason.

The identified 32-aa sequence of NLP1 (NLP1C32) is relatively conserved in other plant-pathogenic fungi and oomycetes, suggesting the possible recognition of the C-terminal region of the NLPs by plants. We also pretreated cucumber with synthetic peptides corresponding to the $\mathrm{C}$-terminal region of CoNLP1 and then inoculated $C$. orbiculare on the treated cucumber. However, we did not find any increased resistance to the pathogen in the pretreated cucumber (data not shown). Thus, it remains to be elucidated whether or not the synthetic peptide of the CoNLP1 C-terminal region is sufficient for defense activation of Cucurbitaceae plants. We cannot exclude the possibility that the tertiary structure of the 32-aa region, including its modification, is necessary for the defense activation. For a detailed understanding of how Cucurbitaceae plants recognize the C-terminal region of NLP1 and mount a strong defense as its output, further studies are essential, including the identification of a corresponding NLP1 receptor in Cucurbitaceae plants.

Collectively, our data indicate different evolutionary processes for NLP recognition among higher plants, for example between members of families Brassicaceae and Cucurbitaceae. The MAMP sequence of bacterial flagellin (flg22) is known to be recognized by a wide range of plants, but, importantly, it was reported that a different region of bacterial flagellin is also recognized by a part of plants, including tomato (Cai et al. 2011; Hind et al. 2016). Thus, our data provides the example that a conserved molecule of fungal and oomycete pathogens is also recognized at distinct positions by higher plants, probably via independent evolution.

Our data also suggests that the strong activation of MAMPtriggered immunity (MTI) is sufficient to block host infection by $C$. orbiculare, especially at the appressorium-mediated hostinvasion step. Consistently, in nonhost interactions, appressoria of Colletotrichum species fail to develop invasion hyphae, e.g., appressoria of $C$. orbiculare never develop invasive hyphae in nonhost A. thaliana (Shimada et al. 2006). This type of phenomenon is also observed in the interaction of susceptible plants with $C$. orbiculare ssdl mutants that can overactivate MTI (Tanaka et al. 2007, 2009). Thus, the strong activation of MTI is sufficient to terminate the penetration function of melanized appressoria of Colletotrichum species that can generate strong turgor pressure (Bechinger et al. 1999).

However, it is also plausible that the WT strain of $C$. orbiculare suppresses the defense of host cucumber by deploying appropriate effectors, whereas the pathogen fails to infect $A$. thaliana because it has no appropriate effectors. The question is why the C. orbiculare transgenic lines expressing NLP1 failed to infect cucumber, even in the presence of an effective effector set. We assume that the putative effector set might not be sufficient to suppress the strong immune responses triggered by the recognition of NLP1 when constitutively expressed. Consistent with this, it has been reported that the overexpression of a glycoside hydrolase family 12 protein, XEG1, recognized as a MAMP, inhibited $P$. sojae infection on soybean (Ma et al. 2015). Based on the genome sequence of $C$. orbiculare, the pathogen has seven NLP genes (Gan et al. 2013). Microarray analysis suggested that multiple $N L P$ genes are likely expressed during the early phases of infection (Gan et al. 2013). We found that the corresponding regions for NLP1C32 in the other NLPs of $C$. orbiculare likely exhibit conservation with CoNLP1 to some degree (Supplementary Fig. S7), but they also represent increased variations in comparison with the CoNLP1 homologs of other fungi and oomycetes. The reason why the expression of the other $C$. orbiculare $N L P$ genes during the early infection phase does not elicit strong defense on cucumber might be that i) the expression level is not enough for the elicitation or ii) their sequence or structure is not recognized by the plant in contrast to NLP1.

\section{MATERIALS AND METHODS}

Fungal strains, media, transformation, and DNA analysis. Colletotrichum orbiculare (syn. C. lagenarium) wild-type strain 104-T (MAFF240422) is stored at the Laboratory of Plant Pathology, Kyoto University. All C. orbiculare strains were maintained on $3.9 \%$ (wt/vol) potato dextrose agar (PDA) (Nissui, Tokyo) at $24^{\circ} \mathrm{C}$ in the dark. The transformation of $C$. orbiculare was based on the method of Kimura et al. (2001). Bialaphosresistant transformants were selected on PDA plates with $25 \mu \mathrm{g}$ of bialaphos per milliliter (Wako Pure Chemicals, Osaka, Japan). The total DNA of C. orbiculare was isolated from mycelia with the DNeasy plant mini kit (Qiagen, Hilden, Germany), according to the manufacturer's instructions.

\section{Plasmid construction.}

All plasmids used for constitutive expression of NLP1 and its derivatives were constructed using the plasmid pBATTEFP (Asakura et al. 2009). pBATTEFP was generated by introduction of the $T E F$ promoter into pBAT (Kimura et al. 2001), a derivative of pCB1531 (Sweigard et al. 1997) that carries the bialaphos resistance gene (bar). All primers used in this study are listed in Supplementary Table S1.

To constitutively express NLP1 in $C$. orbiculare under the control of the TEF promoter, NLPI cDNA fused with a hemagglutinin (HA) epitope tag sequence was amplified with primers TEFpNLP1_f and TEFpNLP1_r. The amplified fragment was digested with $X b a \mathrm{I}$ and $E c o$ RI and was introduced into pBATTEFP to produce pBATTEFPNLP1.

To generate mutant NLP1 lacking its cytotoxic activity, one single site-directed mutagenesis was carried out to substitute His 127 in NLP1 with Ala (CAC to GCA) by recombinant PCR, using the primer pairs TEFpNLP1_f/H127A_r and H127A_f/ TEFpNLP1_r. Then, the full-length fragment of NLP1H127A 
fused with a HA epitope tag was amplified using TEFpNLP1_f and TEFpNLP1_r primers. The amplified fragment of the HA-tagged NLP1H127A was digested with $X b a I$ and EcoRI and was then inserted into pBATTEFP, resulting in pBATTEFPNLP1H127A.

For the construction of mutant NLP1 lacking the functional nlp24 sequence, site-directed mutagenesis by primer extension was performed to substitute Ile111, Met112, and Tyr113 with Ala (ATC ATG TAC to GCC GCG GCC). In the first round of PCR, primer sets NLP1_XbaI_F/NLP1_MAMP_R and NLP1_ MAMP_F/TEFpNLP1_r were used to amplify two products with the mutated sequence at a known MAMP region. In the next round of PCR, NLP1_XbaI_F and TEFpNLP1_r primers were used to amplify the full-length of $N L P 1$ fused with the HA epitope tag, which contained the desired mutation. Next, the amplified fragments of $N L P 1$ fused with the HA epitope tag were digested with $X b a \mathrm{I}$ and EcoRI and were then inserted into pBATTEFP, resulting in pBATTEFPNLP1Mut.

To express HA-tagged NLP1 under the control of the 35S promoter, a BamHI-EcoRI fragment of NLPl was amplified by primers 35SpNLP1_f and 35SpNLP1_r. The amplified fragment was digested with $B a m \mathrm{HI}$ and EcoRI and was then introduced into the BamHI-EcoRI site of pBICP35 (Mori et al. 1991), resulting in pBICP35NLP1. To express HA-tagged NLP1H127A under the control of the $35 \mathrm{~S}$ promoter, site-directed mutagenesis by primer extension was used to substitute His127 with Ala (CAC to GCA) by using the primer pairs 35SpNLP1_f/H127A_r and H127A_f/35SpNLP1_r. Next, 35SpNLP1_f and 35SpNLP1_r primers were used to amplify the full length of $N L P 1 \mathrm{H} 127 \mathrm{~A}$. The amplified fragment was digested with $B a m \mathrm{HI}$ and $E c o \mathrm{RI}$ and was then inserted into the BamHI-EcoRI site of pBICP35, resulting in pBICP35NLP1H127A.

To clarify the importance of the NLP1 signal peptide, a plasmid carrying NLPI lacking the region encoding the signal peptide (NLP1 $\triangle$ SP-HA) was constructed. The NLPl cDNA lacking the signal peptide was amplified with primers TEFpNLPdSP_f and TEFpNLP1_r. The amplified fragment was digested with $X b a \mathrm{I}$ and EcoRI and was introduced into pBATTEFP to produce pBATTEFPNLP1 $\triangle$ SP.

To identify the NLP1 region that is recognized by cucumber, cDNA fragments of $N L P 1 \triangle 22, N L P 1 \triangle 42$, and $N L P 1 \triangle 62$ were amplified by PCR with the following primers: NLP1_XbaI_F/ NLP1_del_217_R for NLP1 22 , NLP1_XbaI_F/ NLP1_del_197_R for $N L P 1 \triangle 42$, and NLP1_XbaI_F/ NLP1_del_177_R for $N L P 1 \triangle 62$. The amplified fragments were digested with $X b a \mathrm{I}$ and EcoRI and were introduced into pBATTEFP, resulting in pBATTEFPNLP1 $\triangle 22$, pBATTEFPNLP1 $\triangle 42$, and pBATTEFPNLP1 $\Delta 62$, respectively.

To investigate whether the carboxy terminal 32-aa region of NLP1 is sufficient to trigger cucumber defense, two expression vectors, pBATTEFPmCNLP1FL and pBATTEFPmCNLP1C32, were constructed by using a multifragment cloning method. $\mathrm{SP}^{\mathrm{CoDN} 3 \mathrm{~A}}$ mCherry was amplified, using pBATTEFPSPCoDN3A mCherry (Irieda et al. 2014) as a template with the first primer set NLP1_Sp_mCherry_F1/NLP1_Sp_mCherry_R1. In parallel, the second primer sets, NLP1_217_mCherry_F2/NLP1_Sp_mCherry_ R2 and NLP1_32_mCherry_F2/NLP1_Sp_mCherry_R2, were designed and used to amplify NLP1 $\triangle \mathrm{SP}$ and 32 amino acids of the carboxy-terminal region of NLP1 fragments, respectively. Each amplified fragment was assembled and inserted into pBATTEFP linearized with $X b a \mathrm{I}$ and $E c o$ RI, using the In-Fusion HD cloning kit (Takara, Otsu, Japan) according to the manufacturer's protocol.

\section{Inoculation assay of $C$. orbiculare.}

In the inoculation assay of $C$. orbiculare on cucumber, $10 \mu \mathrm{l}$ of conidial suspensions $\left(5 \times 10^{5}\right.$ conidia/ml $)$ were drop-inoculated onto detached cucumber cotyledons (Cucumis sativus), melon (Cucumis melo), winter melon (Benincasa hispida), or long melon (Cucumis melo L. var. utilissimus). Inoculated cotyledons were incubated at $24^{\circ} \mathrm{C}$ for 7 days. The cotyledons were picked from 10-day-old plants (cucumber, melon, and long melon) or from 2-week-old plants (winter melon). For inoculation on $N$. benthamiana, true leaves detached from approximately 5-week-old plants were used. Each virulence test was performed at least three times under the same conditions.

\section{Light microscopy.}

To investigate appressorium-mediated host invasion by C. orbiculare or detect ROS accumulation, 10- $\mu$ l conidial suspensions $\left(5 \times 10^{5}\right.$ conidia per milliliter of distilled water $)$ were spotted onto the abaxial surface of cucumber cotyledons and were incubated in a humid chamber at $24^{\circ} \mathrm{C}$. For appressoriummediated host invasion, we peeled off the lower epidermis of the cotyledons at 4 days postinoculation (dpi) and placed them on glass slides for observation. The invasive hyphae were observed in bright field by using an Olympus BX53 fluorescence microscope (Olympus, Tokyo) equipped with an Olympus DP72 camera and Olympus cellSens software (Lin et al. 2012).

For the detection of ROS accumulation, the abaxial surface of cucumber cotyledons at $2 \mathrm{dpi}$ was soaked in a $1 \mathrm{mg} / \mathrm{ml}$ DAB (Santa Cruz Biotechnology, Dallas, TX, U.S.A.) solution for $24 \mathrm{~h}$ at $24^{\circ} \mathrm{C}$ in the dark (Fukada and Kubo 2015). Then, the abaxial surfaces of stained cotyledons were peeled off and were observed with a bright field Olympus BX53 fluorescence microscope equipped with an Olympus DP72 camera and Olympus cellSens software.

\section{A. tumefaciens-mediated transient expression in $N$. benthamiana.}

$N$. benthamiana plants (5 weeks old) were used for the agroinfiltration assay. Plants were grown in a controlled environment chamber at $25^{\circ} \mathrm{C}$ with $16 \mathrm{~h}$ of illumination per day. Each construct (pBICP35NLP1, pBICP35NLP1H127A) was transformed into Agrobacterium tumefaciens GV3101 pMP90 by electroporation. Each transformant was cultured in yeast extract peptone media containing kanamycin $(100 \mu \mathrm{g} / \mathrm{ml})$, rifampicin $(100 \mu \mathrm{g} / \mathrm{ml})$, and gentamycin $(50 \mu \mathrm{g} / \mathrm{ml})$. The cells were harvested by centrifugation and were suspended in MMA induction buffer (1 liter of MMA: $5 \mathrm{~g}$ of Murashige-Skoog salts, $1.95 \mathrm{~g}$ of MES, $20 \mathrm{~g}$ of sucrose, and $200 \mu \mathrm{M}$ acetosyringone, $\mathrm{pH}$ 5.6) (Yoshino et al. 2012). All suspensions were incubated for 1 to $3 \mathrm{~h}$ at room temperature prior to infiltration. The suspensions were infiltrated into $N$. benthamiana leaves using a syringe. The infiltrated areas were observed at 5 days after the infiltration.

\section{Detection of oxidative burst in leaf discs of $A$. thaliana.}

ROS released by leaf tissue was assayed as described (Ranf et al. 2011), using 3-mm leaf discs in 96-well plates containing $0.1 \mathrm{ml}$ of distilled water supplied with $20 \mu \mathrm{M}$ luminal and $1 \mu \mathrm{g}$ of horseradish peroxidase (Sigma-Aldrich, St. Louis), measured in 2-min intervals for $40 \mathrm{~min}$, using Luminoskan Ascent 2.1. (Thermo Scientific, Yokohama, Japan).

\section{qRT-PCR analysis.}

Cucumber cotyledons were drop-inoculated with $10-\mu \mathrm{l}$ conidial suspensions $\left(5 \times 10^{5}\right.$ conidia $\left./ \mathrm{ml}\right)$ and were incubated for 1 day in a humid chamber at $24^{\circ} \mathrm{C}$. Total RNA was extracted, using the RNeasy plant mini kit (Qiagen) according to the manufacture's protocol, and was treated with Promega RQ1 RNasefree DNase (Promega, Madison, WI, U.S.A.) to remove DNA contamination. The first-strand cDNA was synthesized using Takara PrimeScript RT reagent kit (Takara), followed by qPCR with specific primers to nucleotide sequences corresponding to 32 aa of the NLP1 C-terminal region. The expression of the actin gene of $C$. orbiculare was used as an internal standard. The qPCR 
was carried out using SYBR premix ExTaq II (Takara) with a Thermal Cycler Dice real time system TP800 (Takara).

\section{ACKNOWLEDGMENTS}

We thank Y. Kubo (Kyoto Prefectural University, Japan) for $C$. orbiculare cst1 mutant RCS2-1. This work was supported in part by Grants-in-Aid for Scientific Research (15H05780 and 15H04457) from the Ministry of Education, Culture, Sports, Science and Technology of Japan, by an Institution for Fermentation, Osaka (IFO) research grant, and by the Science and Technology Research Promotion Program for Agriculture, Forestry, Fisheries and Food industry.

\section{LITERATURE CITED}

Albert, I., Böhm, H., Albert, M., Feiler, C. E., Imkampe, J., Wallmeroth, N., Brancato, C., Raaymakers, T. M., Oome, S., Zhang, H., Krol, E., Grefen, C., Gust, A. A., Chai, J., Hedrich, R., Van den Ackerveken, G., and Nürnberger, T. 2015. An RLP23-SOBIR1-BAK1 complex mediates NLP-triggered immunity. Nat Plants 1:15140.

Amsellem, Z., Cohen, B. A., and Gressel, J. 2002. Engineering hypervirulence in a mycoherbicidal fungus for efficient weed control. Nat. Biotechnol. 20:1035-1039.

Asakura, M., Ninomiya, S., Sugimoto, M., Oku, M., Yamashita, S., Okuno, T., Sakai, Y., and Takano, Y. 2009. Atg26-mediated pexophagy is required for host invasion by the plant pathogenic fungus Colletotrichum orbiculare. Plant Cell 21:1291-1304.

Bae, H., Kim, M. S., Sicher, R. C., Bae, H. J., and Bailey, B. A. 2006. Necrosis- and ethylene-inducing peptide from Fusarium oxysporum induces a complex cascade of transcripts associated with signal transduction and cell death in Arabidopsis. Plant Physiol. 141:1056-1067.

Bailey, B. A. 1995. Purification of a protein from culture filtrates of Fusarium oxysporum that induces ethylene and necrosis in leaves of Erythroxylum coca. Phytopathology 85:1250-1255.

Bechinger, C., Giebel, K. F., Schnell, M., Leiderer, P., Deising, H. B., and Bastmeyer, M. 1999. Optical measurements of invasive forces exerted by appressoria of a plant pathogenic fungus. Science 285:1896-1899.

Böhm, H., Albert, I., Oome, S., Raaymakers, T. M., Van den Ackerveken, G., and Nürnberger, T. 2014. A conserved peptide pattern from a widespread microbial virulence factor triggers pattern-induced immunity in Arabidopsis. PLoS Pathog. 10:e1004491.

Boller, T., and Felix, G. 2009. A renaissance of elicitors: Perception of microbe-associated molecular patterns and danger signals by patternrecognition receptors. Annu. Rev. Plant Biol. 60:379-406.

Cabral, A., Oome, S., Sander, N., Küfner, I., Nürnberger, T., and Van den Ackerveken, G. 2012. Nontoxic Nep1-like proteins of the downy mildew pathogen Hyaloperonospora arabidopsidis: repression of necrosis-inducing activity by a surface-exposed region. Mol. Plant-Microbe Interact. 25: 697-708.

Cai, R., Lewis, J., Yan, S., Liu, H., Clarke, C. R., Campanile, F., Almeida, N. F., Studholme, D. J., Lindeberg, M., Schneider, D., Zaccardelli, M., Setubal, J. C., Morales-Lizcano, N. P., Bernal, A., Coaker, G., Baker, C., Bender, C. L., Leman, S., and Vinatzer, B. A. 2011. The plant pathogen Pseudomonas syringae pv. tomato is genetically monomorphic and under strong selection to evade tomato immunity. PLoS Pathog. 7: e1002130.

Dong, S., Kong, G., Qutob, D., Yu, X., Tang, J., Kang, J., Dai, T., Wang, H., Gijzen, M., and Wang, Y. 2012. The NLP toxin family in Phytophthora sojae includes rapidly evolving groups that lack necrosis-inducing activity. Mol. Plant-Microbe Interact. 25:896-909.

Fukada, F., and Kubo, Y. 2015. Colletotrichum orbiculare regulates cell cycle G1/S progression via a two-component GAP and a GTPase to establish plant infection. Plant Cell 27:2530-2544.

Gan, P., Ikeda, K., Irieda, H., Narusaka, M., O'Connell, R. J., Narusaka, Y., Takano, Y., Kubo, Y., and Shirasu, K. 2013. Comparative genomic and transcriptomic analyses reveal the hemibiotrophic stage shift of Colletotrichum fungi. New Phytol. 197:1236-1249.

Gijzen, M., and Nürnberger, T. 2006. Nep1-like proteins from plant pathogens: Recruitment and diversification of the NPP1 domain across taxa. Phytochemistry 67:1800-1807.

Hind, S. R., Strickler, S. R., Boyle, P. C., Dunham, D. M., Bao, Z., O’Doherty, I. M., Baccile, J. A., Hoki, J. S., Viox, E. G., Clarke, C. R., Vinatzer, B. A., Schroeder, F. C., and Martin, G. B. 2016. Tomato receptor FLAGELLIN-SENSING 3 binds flgII-28 and activates the plant immune system. Nat. Plants 2:16128.

Irieda, H., Maeda, H., Akiyama, K., Hagiwara, A., Saitoh, H., Uemura, A., Terauchi, R., and Takano, Y. 2014. Colletotrichum orbiculare secretes virulence effectors to a biotrophic interface at the primary hyphal neck via exocytosis coupled with SEC22-mediated traffic. Plant Cell 26:2265-2281.

Kimura, A., Takano, Y., Furusawa, I., and Okuno, T. 2001. Peroxisomal metabolic function is required for appressorium-mediated plant infection by Colletotrichum lagenarium. Plant Cell 13:1945-1957.

Kleemann, J., Rincon-Rivera, L. J., Takahara, H., Neumann, U., Ver Loren van Themaat, E., van der Does, H. C., Hacquard, S., Stüber, K., Will, I., Schmalenbach, W., Schmelzer, E., and O'Connell, R. J. 2012. Sequential delivery of host-induced virulence effectors by appressoria and intracellular hyphae of the phytopathogen Colletotrichum higginsianum. PLoS Pathog. 8:e1002643.

Kubo, Y., and Takano, Y. 2013. Dynamics of infection-related morphogenesis and pathogenesis in Colletotrichum orbiculare. J. Gen. Plant Pathol. 79:233-242.

Lin, S. Y., Okuda, S., Ikeda, K., Okuno, T., and Takano, Y. 2012. LAC2 encoding a secreted laccase is involved in appressorial melanization and conidial pigmentation in Colletotrichum orbiculare. Mol. Plant-Microbe Interact. 25:1552-1561

Ma, Z., Song, T., Zhu, L., Ye, W., Wang, Y., Shao, Y., Dong, S., Zhang, Z., Dou, D., Zheng, X., Tyler, B. M., and Wang, Y. 2015. A Phytophthora sojae glycoside hydrolase 12 protein is a major virulence factor during soybean infection and is recognized as a PAMP. Plant Cell 27:2057-2072.

Mori, M., Mise, K., Kobayashi, K., Okuno, T., and Furusawa, I. 1991. Infectivity of plasmids containing brome mosaic virus cDNA linked to the cauliflower mosaic virus 35S RNA promoter. J. Gen. Virol. 72:243-246.

O'Connell, R. J., Thon, M. R., Hacquard, S., Amyotte, S. G., Kleemann, J., Torres, M. F., Damm, U., Buiate, E. A., Epstein, L., Alkan, N., Altmüller, J., Alvarado-Balderrama, L., Bauser, C. A., Becker, C., Birren, B. W., Chen, Z., Choi, J., Crouch, J. A., Duvick, J. P., Farman, M. A., Gan, P., Heiman, D., Henrissat, B., Howard, R. J., Kabbage, M., Koch, C., Kracher, B., Kubo, Y., Law, A. D., Lebrun, M. H., Lee, Y. H., Miyara, I., Moore, N., Neumann, U., Nordström, K., Panaccione, D. G., Panstruga, R., Place, M., Proctor, R. H., Prusky, D., Rech, G., Reinhardt, R., Rollins, J. A., Rounsley, S., Schardl, C. L., Schwartz, D. C., Shenoy, N., Shirasu, K., Sikhakolli, U. R., Stüber, K., Sukno, S. A., Sweigard, J. A., Takano, Y., Takahara, H., Trail, F., van der Does, H. C., Voll, L. M., Will, I., Young, S., Zeng, Q., Zhang, J., Zhou, S., Dickman, M. B., SchulzeLefert, P., Ver Loren van Themaat, E., Ma, L. J., and Vaillancourt, L. J. 2012. Lifestyle transitions in plant pathogenic Colletotrichum fungi deciphered by genome and transcriptome analyses. Nat. Genet. 44: 1060-1065

Oome, S., Raaymakers, T. M., Cabral, A., Samwel, S., Böhm, H., Albert, I., Nürnberger, T., and Van den Ackerveken, G. 2014. Nep1-like proteins from three kingdoms of life act as a microbe-associated molecular pattern in Arabidopsis. Proc. Natl. Acad. Sci. U.S.A. 111:16955-16960.

Oome, S., and Van den Ackerveken, G. 2014. Comparative and functional analysis of the widely occurring family of Nep1-like proteins. Mol. Plant-Microbe Interact. 27:1081-1094.

Ottmann, C., Luberacki, B., Küfner, I., Koch, W., Brunner, F., Weyand, M., Mattinen, L., Pirhonen, M., Anderluh, G., Seitz, H. U., Nürnberger, T., and Oecking, C. 2009. A common toxin fold mediates microbial attack and plant defense. Proc. Natl. Acad. Sci. U.S.A. 106:10359-10364.

Perfect, S. E., Hughes, H. B., O'Connell, R. J., and Green, J. R. 1999. Colletotrichum: A model genus for studies on pathology and fungalplant interactions. Fungal Genet. Biol. 27:186-198.

Qutob, D., Kamoun, S., and Gijzen, M. 2002. Expression of a Phytophthora sojae necrosis-inducing protein occurs during transition from biotrophy to necrotrophy. Plant J. 32:361-373.

Qutob, D., Kemmerling, B., Brunner, F., Küfner, I., Engelhardt, S., Gust, A. A., Luberacki, B., Seitz, H. U., Stahl, D., Rauhut, T., Glawischnig, E., Schween, G., Lacombe, B., Watanabe, N., Lam, E., Schlichting, R., Scheel, D., Nau, K., Dodt, G., Hubert, D., Gijzen, M., and Nürnberger, T. 2006. Phytotoxicity and innate immune responses induced by Nep1-like proteins. Plant Cell 18:3721-3744.

Ranf, S., Eschen-Lippold, L., Pecher, P., Lee, J., and Scheel, D. 2011. Interplay between calcium signalling and early signalling elements during defence responses to microbe- or damage-associated molecular patterns. Plant J. 68:100-113.

Shen, S., Goodwin, P. H., and Hsiang, T. 2001. Infection of Nicotiana species by the anthracnose fungus, Colletotrichum orbiculare. Eur. J. Plant Pathol. 107:767-773.

Shimada, C., Lipka, V., O'Connell, R., Okuno, T., Schulze-Lefert, P., and Takano, Y. 2006. Nonhost resistance in Arabidopsis-Colletotrichum interactions acts at the cell periphery and requires actin filament function. Mol. Plant-Microbe Interact. 19:270-279.

Staats, M., VAN Baarlen, P., Schouten, A., and VAN Kan, J. A. L. 2007. Functional analysis of NLP genes from Botrytis elliptica. Mol. Plant Pathol. 8:209-214. 
Sweigard, J., Chumley, F., Carroll, A., Farrall, L., and Valent, B. 1997. A series of vectors for fungal transformation. Fungal Genet. Newsl. 44:52-55.

Takano, Y., Takayanagi, N., Hori, H., Ikeuchi, Y., Suzuki, T., Kimura, A., and Okuno, T. 2006. A gene involved in modifying transfer RNA is required for fungal pathogenicity and stress tolerance of Colletotrichum lagenarium. Mol. Microbiol. 60:81-92.

Tanaka, S., Ishihama, N., Yoshioka, H., Huser, A., O’Connell, R., Tsuji, G., Tsuge, S., and Kubo, Y. 2009. The Colletotrichum orbiculare SSD1 mutant enhances Nicotiana benthamiana basal resistance by activating a mitogen-activated protein kinase pathway. Plant Cell 21:2517-2526.

Tanaka, S., Yamada, K., Yabumoto, K., Fujii, S., Huser, A., Tsuji, G., Koga, H., Dohi, K., Mori, M., Shiraishi, T., O'Connell, R., and Kubo, Y. 2007. Saccharomyces cerevisiae SSD1 orthologues are essential for host infection by the ascomycete plant pathogens Colletotrichum lagenarium and Magnaporthe grisea. Mol. Microbiol. 64:1332-1349.

Tsuji, G., Fujii, S., Tsuge, S., Shiraishi, T., and Kubo, Y. 2003. The Colletotrichum lagenarium Ste12-like gene CST1 is essential for appressorium penetration. Mol. Plant-Microbe Interact. 16:315-325.

Vanden Wymelenberg, A. J., Cullen, D., Spear, R. N., Schoenike, B., and Andrews, J. H. 1997. Expression of green fluorescent protein in Aureobasidium pullulans and quantification of the fungus on leaf surfaces. Biotechniques 23:686-690.

Yoshino, K., Irieda, H., Sugimoto, F., Yoshioka, H., Okuno, T., and Takano, Y. 2012. Cell death of Nicotiana benthamiana is induced by secreted protein NIS1 of Colletotrichum orbiculare and is suppressed by a homologue of $C g D N 3$. Mol. Plant-Microbe Interact. 25:625-636. 